\title{
EXIGÊNCIAS NUTRICIONAIS DA GRÁPIA (Apuleia leiocarpa Vog. Macbride) EM SOLO PODZÓLICO VERMELHO AMARELO ${ }^{1}$
}

\author{
NUTRITIONAL REQUIREMENTS OF GRÁPIA (Apuleia leiocarpa \\ Vog. Macbride) IN A RED YELLOW PODZOLIC SOIL
}

\author{
Fernando Teixeira Nicoloso ${ }^{2}$ Flavio Zanchetti ${ }^{3}$ \\ Alencar Garlet ${ }^{3}$ Marco Aurélio de Freitas Fogaça ${ }^{4}$
}

RESUMO

A grápia (Apuleia leiocarpa Vog. Macbride) é uma espécie florestal nativa que se encontra atualmente em processo de extinção. $O$ presente trabalho foi desenvolvido com o objetivo de caracterizar as exigências nutricionais da grápia pela técnica do nutriente faltante em solo Podzólico vermelho amarelo (unidade de mapeamento São Pedro). As plantas de grápia foram cultivadas em vasos contendo solo, sob condições de casa de vegetação. Foram testados 18 tratamentos em esquema fatorial (2 X 9), onde os fatores consistiram em dois horizontes de um mesmo solo (A e B) e 9 variações de adubação. Os tratamentos de adubação foram: (1) solo natural; (2) adubação completa com macro e micronutrientes (completo); (3) completo $-N$; (4) completo $-\mathrm{P}$; (5) completo $-\mathrm{K}$; (6) completo $-\mathrm{Ca}$; (7) completo $-\mathrm{Mg}$; (8) completo - S e (9) completo - Zn, Cu, Fe, Mn, B e Mo. Após 165 dias de cultivo, avaliaram-se a produção de massa seca da parte aérea, do sistema radicular e total da planta, relação entre massa seca de raiz e parte aérea, número de folhas remanescentes, comprimento total do sistema radicular, diâmetro do caule, altura da planta, número de nós e os teores de $\mathrm{N}, \mathrm{P}, \mathrm{K}, \mathrm{Ca}, \mathrm{Mg}$, $\mathrm{Zn}, \mathrm{Cu}, \mathrm{Mn}$ e Fe nos órgãos da planta. A omissão isolada de Ca, $\mathrm{Mg}$ e dos micronutrientes $\mathrm{Zn}, \mathrm{Cu}, \mathrm{Fe}, \mathrm{Mn}$ da adubação não afeta significativamente o crescimento da grápia em altura, diâmetro do caule, número de nós, número de folhas e a produção da massa seca, bem como a concentração desses elementos nos tecidos da planta, demonstrando que os teores naturais desses nutrientes no solo são suficientes. A altura da planta, número de nós, número de folhas, comprimento do sistema radicular, produção de massa seca e a concentração de nutrientes diminuem acentuadamente pela omissão de $P, S, N$ e $K$ da adubação, tanto no horizonte A como no B do solo.
Palavras-chave: grápia, nutrição mineral, adubação, Apuleia leiocarpa, nutriente faltante, podzólico vermelho amarelo.

SUMMARY

Grápia (Apuleia leiocarpa Vog. Macbride) is a native forest specie that has been in extinction process. The purpose of this study was to characterize the nutritional requirements of grápia in a red yellow podzolic soil by using the lacking nutrient technique. Grápia plants were cultivated in pots containing soil, under glasshouse conditions. Eighteen treatments were tested in a fatorial design ( 2 X 9). The factors consisted of two profiles of the same soil $(A$ and $B$ ) and nine fertilization treatments. The fertilization treatments were: (1) natural soil, (2) complete fertilization with macro and micronutrients (complete), (3) complete less $N$, (4) complete less $P$, (5) complete less $K,(6)$ complete less $C a$, (7) complete less $M g$, (8) complete less $S$, and (9) complete less the micronutrients $\mathrm{Zn}, \mathrm{Cu}, \mathrm{Fe}, \mathrm{Mn}, \mathrm{B}$ and $\mathrm{Mo}$. After 165 days of cultivation the growth parameters analysed were dry weight of roots and aerial part, relationship between dry weight of root and aerial part, number of leaves, total lenght of the root system, shoot diameter, height of the plant, number of shoot nodes, and the concentration of $\mathrm{N}, \mathrm{P}, \mathrm{K}, \mathrm{Ca}, \mathrm{Mg}, \mathrm{Zn}, \mathrm{Cu}$, $\mathrm{Mn}$ and $\mathrm{Fe}$ in the plants parts. The isolated omission of $\mathrm{Ca}, \mathrm{Mg}$, and of the micronutrients $\mathrm{Zn}, \mathrm{Cu}, \mathrm{Fe}$ and Mn from the fertilization do not affect the growth of grápia in height, shoot diameter, number of shoot nodes, number of leaves, and biomass accumulation, as well as the nutrient concentration in the plant tissues, indicating that the natural nutrient levels are adequate. The height of the plant, number of shoot nodes, number of leaves, total lenght of the root system, biomass accumulation and the

\footnotetext{
${ }^{1}$ Projeto financiado pela Fundação de Apoio à Pesquisa e Extensão (FIPE) - Universidade Federal de Santa Maria (UFSM) e FAPERGS. ${ }^{2}$ Engenheiro Agrônomo, PhD., Professor Adjunto do Departamento de Biologia, Centro de Ciências Naturais e Exatas (CCNE), UFSM, 97105-900, Santa Maria, RS. E-mail: nicoloso@sm.conex.com.br. Autor para correspondência.

${ }^{3}$ Acadêmico do curso de Engenharia Florestal, UFSM, bolsista do CNPq.

${ }^{4}$ Engenheiro Agrônomo, Mestrando em Agronomia, UFSM, bolsista da CAPES.

Recebido para publicação em 27.05.98. Aprovado em 09.09.98
} 
nutrient concentration decrease strikingly by omitting $P, S, N$ and $K$ from the fertilization, for both profiles of the soil.

Key words: grápia, mineral nutrition, fertilization, Apuleia leiocarpa, nutrient absence, red yellow podzolic.

\section{INTRODUÇÃO}

A grápia (Apuleia leiocarpa Vog. Macbride) é uma planta leguminosa que tem uma distribuição muito ampla no território brasileiro. Entretanto, essa espécie torna-se cada vez mais escassa devido à devastação das florestas na sua área de ocorrência natural e à exploração de sua madeira de forma extrativista, sem haver reposição através de reflorestamento. É uma planta de elevado porte, atingindo grandes dimensões, portanto de interesse madeireiro. A sua madeira tem usos múltiplos e a sua casca chega a ter $24 \%$ de taninos, servindo para a indústria de curtumes.

É uma espécie de caráter pioneiro, notando-se não haver pronunciada afinidade por determinadas condições físicas de solo. De modo geral, a espécie é mais abundante em solos com rápida drenagem, podendo ocorrer também em regiões de planalto, em solos secos e de baixa fertilidade (HERINGER \& FERREIRA, 1973). Um dos grandes problemas na elaboração de programas de plantios florestais, principalmente com árvores nativas, é o escasso conhecimento acerca da auto-ecologia das espécies (Kageyama apud BRAGA et al., 1995). Neste âmbito, pode-se ressaltar a falta absoluta de informações sobre as exigências nutricionais da grápia.

Em razão das diferentes condições de ocorrência natural dessa espécie, espera-se que sua exigência nutricional seja distinta das demais espécies cultivadas, o que levaria, no caso de plantios artificiais, à escolha de solo com fertilidade diferenciada ou diferentes recomendações de adubação e correção para um mesmo tipo de solo.

$\mathrm{Na}$ avaliação de exigências nutricionais, o uso da técnica do nutriente faltante é uma dentre outras que melhor se adapta ao propósito, tendo sido utilizada sob condições de casa de vegetação e tendo solo como substrato para Araucaria angustifolia (SIMÕES, 1973), Pinus caribae (MARTINEZ et al., 1992), Cyperus malaccensis (HIROCE et al., 1990) e Boehmeria nivea (FEITOSA et al., 1992).

O presente estudo foi desenvolvido com objetivo de caracterizar as exigências nutricionais da grápia pela técnica do nutriente faltante em solo Podzólico vermelho amarelo.

\section{MATERIAL E MÉTODOS}

O experimento foi instalado em 14 de janeiro de 1997, na casa de vegetação do Departamento de Fitotecnia da Universidade Federal de Santa Maria (UFSM), Santa Maria - RS. Foram testados 18 tratamentos em esquema fatorial $(2 \times 9)$, onde os fatores consistiram em dois horizontes (A e B) de um mesmo solo e nove variações na adubação, com quatro repetições, perfazendo 72 parcelas experimentais, num delineamento inteiramente casualizado. Cada parcela constituiu-se de um vaso, com $4,0 \mathrm{~kg}$ de terra fina seca ao ar e duas plantas, os quais foram submetidos a rodízio periódico para evitar efeito local.

Os tratamentos de adubação foram: (1) solo natural, (2) adubação completa com macro e micronutrientes (completo), (3) completo -N, (4) completo -P, (5) completo -K, (6) completo - $\mathrm{Ca}$, (7) completo - $\mathrm{Mg}$, (8) completo -S e (9) completo - Zn, $\mathrm{Cu}, \mathrm{Fe}, \mathrm{Mn}, \mathrm{B}$ e Mo. A aplicação dos nutrientes foi realizada através de soluções aquosas. A adubação completa consistiu em: $\mathrm{N}=150,0 ; \mathrm{P}=100,0 ; \mathrm{K}=$ 40,$0 ; \mathrm{Ca}=40,0 ; \mathrm{Mg}=20,0 ; \mathrm{S}=30,0 ; \mathrm{Zn}=1,0 ; \mathrm{B}=$ 0,$25 ; \mathrm{Cu}=0,10 ; \mathrm{Fe}=0,50 ; \mathrm{Mn}=0,10$ e $\mathrm{Mo}=$ $0,05 \mathrm{mgkg}^{-1}$ de solo, e as dos tratamentos de omissão concentrações idênticas, exceto quanto ao nutriente omitido. As fontes de nutrientes foram: $\mathrm{NH}_{4} \mathrm{H}_{2} \mathrm{PO}_{4}$, $\mathrm{KH}_{2} \mathrm{PO}_{4}, \mathrm{NaH}_{2} \mathrm{PO}_{4}, \mathrm{KCl}, \mathrm{MgSO}_{4} .7 \mathrm{H}_{2} \mathrm{O}, \mathrm{NaSO}_{4}$, $\mathrm{CaCl}_{2}, \mathrm{Ca}\left(\mathrm{H}_{2} \mathrm{PO}_{4}\right)_{2} \cdot \mathrm{H}_{2} \mathrm{O}, \mathrm{KNO}_{3}, \mathrm{Ca}\left(\mathrm{NO}_{3}\right)_{2} \cdot 4 \mathrm{H}_{2} \mathrm{O}$, $\mathrm{NH}_{4} \mathrm{NO}_{3}, \quad \mathrm{MgCl}_{2} \cdot 6 \mathrm{H}_{2} \mathrm{O}, \quad \mathrm{ZnCl}_{2}, \quad \mathrm{H}_{3} \mathrm{BO}_{3}$, $\mathrm{CuSO}_{4} .5 \mathrm{H}_{2} \mathrm{O}, \mathrm{FeSO}_{4} .7 \mathrm{H}_{2} \mathrm{O}$-EDTA, $\mathrm{MnCl}_{2} \cdot 4 \mathrm{H}_{2} \mathrm{O}$ e $\mathrm{H}_{2} \mathrm{MoO}_{4} \cdot \mathrm{H}_{2} \mathrm{O}$. Os nutrientes foram aplicados em dose única no momento da semeadura, exceto o nitrogênio que foi parcelado, $60 \%$ aplicado na semeadura e $40 \%$ em cobertura, aplicado em forma de $\mathrm{NH}_{4} \mathrm{NO}_{3}, 70$ dias após o início do experimento.

As amostras do solo utilizado no experimento foram coletadas de 0 a $65 \mathrm{~cm}$ de profundidade do horizonte $\mathrm{A}$ e de 130 a $180 \mathrm{~cm}$ de profundidade do horizonte $\mathrm{B}$, da unidade de mapeamento São Pedro (Podzólico vermelho amarelo), no município de Santa Maria. Os resultados das análises físicas e químicas, na condição natural e duas semanas após receberem o tratamento de adubação completa são mostrados na tabela 1 .

Os vasos foram forrados internamente com sacos plásticos para evitar a perda de água e nutrientes pela drenagem. Para reposição da água evapotranspirada foi feita irrigação sobre a superfície ou através de dois canos de PVC perfurados e introduzidos no solo. A irrigação foi feita com água destilada, procurando-se manter a umidade do solo entre 40 e $60 \%$ da capacidade de campo, sempre por meio de aferições diárias por pesagem.

As sementes receberam escarificação química, segundo o método descrito por NICOLOSO et al. (1997), seguido de tratamento com solução fungicida à base de Benlate a $0,2 \%$. Após procedeu-se à semeadura de quatro sementes 
Tabela 1 - Resultados das análises química e física do solo Podzólico vermelho amarelo, dos horizontes A e B, antes e após a adubação completa com macro e micronutrientes. UFSM, Santa Maria, 1998.

\begin{tabular}{|c|c|c|c|c|}
\hline \multirow{2}{*}{ Parâmetro } & \multicolumn{2}{|c|}{ Horizonte A } & \multicolumn{2}{|c|}{ Horizonte B } \\
\hline & Solo natural & Solo + adubação & Solo natural & Solo + adubação \\
\hline $\operatorname{argila}(\%)^{(1)}$ & 11,0 & 11,0 & 25,0 & 25,0 \\
\hline mat. Org. $\left(\%{ }^{(2)}\right.$ & 0,7 & 0,7 & 0,1 & 0,1 \\
\hline $\mathrm{pH} . \mathrm{H}_{2} \mathrm{O}^{(3)}$ & 4,8 & 4,5 & 4,7 & 4,0 \\
\hline pH SMP & 5,4 & 5,7 & 4,7 & 5,1 \\
\hline $\mathrm{P}(\mathrm{mg} / \mathrm{L})^{(4)}$ & 8,0 & 62,0 & 2,5 & 35,3 \\
\hline $\mathrm{K}(\mathrm{mg} / \mathrm{L})^{(4)}$ & 16,0 & 60,0 & 12,0 & 54,0 \\
\hline $\mathrm{Ca}\left(\mathrm{Cmol}_{0} / \mathrm{L}\right)^{(5)}$ & 1,0 & 1,0 & 1,7 & 1,9 \\
\hline $\mathrm{Mg}\left(\mathrm{Cmol}_{0} / \mathrm{L}\right)^{(5)}$ & 0,6 & 0,6 & 0,9 & 1,0 \\
\hline $\mathrm{Al}\left(\mathrm{Cmol}_{0} / \mathrm{L}\right)^{(5)}$ & 1,5 & 1,0 & 3,0 & 2,5 \\
\hline $\mathrm{H}+\mathrm{Al}\left(\mathrm{Cmol}_{0} / \mathrm{L}\right)^{(6)}$ & 6,1 & 4,7 & 11,5 & 8,0 \\
\hline $\mathrm{Zn}(\mathrm{mg} / \mathrm{L})^{(7)}$ & 1,6 & 1,6 & 0,5 & 2,3 \\
\hline $\mathrm{Mn}(\mathrm{mg} / \mathrm{L})^{(8)}$ & 10,6 & 15,3 & 5,1 & 7,7 \\
\hline $\mathrm{CTC}\left(\mathrm{Cmol}_{0} / \mathrm{L}\right)^{(9)}$ & 3,5 & 2,8 & 5,6 & 5,5 \\
\hline Sat. Bases (\%) & 25,0 & 27,0 & 19,0 & 27,0 \\
\hline Sat. $\mathrm{Al}(\%)$ & 43,0 & 36,0 & 53,0 & 45,0 \\
\hline
\end{tabular}

(1)- método do densímetro; (2) - oxidação em solução sulfocrômica a quente e determinação por espectofotometria com $\mathrm{Cr}^{+3}$; (3) - relação $1: 1 ;$ (4) - K e P, extrator $\mathrm{HCl} 0,05 \mathrm{~N}+$ $\mathrm{H}_{2} \mathrm{SO}_{4} 0,025 \mathrm{~N}$; (5) - Ca, $\mathrm{Mg}$ e Al, extrator $\mathrm{KCl} 1 \mathrm{~N} ;(6)-\mathrm{H}+\mathrm{Al}$, extrator $\mathrm{CaOAc} 1 \mathrm{~N}$ a $\mathrm{pH}=7$; (7) - Zn, extrator $\mathrm{HCl} 0,1 \mathrm{~N}$; (8) - Mn, extrator $\mathrm{KCl} 1 \mathrm{~N}+\mathrm{HCl}$

teores adequados desses nutrientes para o crescimento da grápia. $\mathrm{O}$ fato de o horizonte $\mathrm{A}$ apresentar-se superior ao B, em quase todos os parâmetros avaliados (tabela 2), talvez reflete-se na maior capacidade desse solo em suprir $\mathrm{P}$ e $\mathrm{K}$ às plantas (tabela 1) e, possivelmente, $\mathrm{N}$ e $\mathrm{S}$, considerando-se que a maior fonte para esses nutrientes é a matéria orgânica (MALAVOLTA, 1984; MENGEL \& KIRKBY, 1979). Por outro lado, pela análise química do solo, verifica-se que os nutrientes $\mathrm{Ca}$ e $\mathrm{Mg}$ apresentam-se em maior concentração no horizonte $\mathrm{B}$ que no A (tabela 1). Uma explicação para justificar o melhor desenvolvimento das plantas no horizonte $\mathrm{A}$ estaria ligado à saturação de $\mathrm{Al}^{+3}$, que no horizonte $\mathrm{B}$ é correspondente a 53\%, ou seja, $10 \%$ maior que aquela do horizonte A. Portanto, os dados sugerem que as plantas submetidas a um maior estresse de $\mathrm{Al}^{+3}$

diretamente nos vasos. Após a emergência das plântulas, fez-se uma seleção das mesmas, deixandose duas plantas uniformes.

Para análise do crescimento das plantas, procedeu-se a uma avaliação aos 165 dias após a semeadura, através dos seguintes parâmetros: produção de massa seca da parte aérea, do sistema radicular e total da planta, relação entre a massa seca do sistema radicular/parte aérea, número de folhas remanescentes, número de nós e altura do caule principal, diâmetro do caule a $1 \mathrm{~cm}$ do solo, comprimento total do sistema radicular, segundo o método descrito por TENNANT (1975), e os teores de N, P, $\mathrm{K}, \mathrm{Ca}, \mathrm{Mg}, \mathrm{Zn}, \mathrm{Cu}, \mathrm{Mn}$ e Fe presentes nos órgãos da planta, segundo TEDESCO et al. (1995). A análise estatística dos parâmetros de crescimento foi realizada pelo teste de Tukey, em nível de probabilidade de erro de $5 \%$.

\section{RESULTADOS E DISCUSSÃO}

\section{Desenvolvimento das plantas:}

A avaliação de diversos parâmetros do desenvolvimento das plantas demonstrou que a omissão de $\mathrm{Ca}, \mathrm{Mg}$ e micronutrientes não alterou significativamente o crescimento (tabela 2), sugerindo que o solo, independente do horizonte, apresenta sofrem distúrbios fisiológicos, que resultam em menor crescimento. Alguns autores (CEVENÁ \& KULHAVÝ, 1991) têm sugerido que o $\mathrm{Al}^{+3}$ deslocaria o $\mathrm{Ca}^{+2}$ das membranas celulares e, assim, desencadearia várias alterações no metabolismo vegetal. Esperava-se, também, que o comprimento total do sistema radicular diferisse nas plantas cultivadas nesses dois horizontes, como esse fato não foi constatado fica indicado uma razoável capacidade da espécie em suportar altas concentrações de $\mathrm{Al}^{+3}$.

NOVAIS et al. (1992) observaram crescimento normal de Eucalyptus grandis e E. saligna em solos contendo 0,40 meq/100g de $\mathrm{Ca}+\mathrm{Mg}$ trocáveis. A concentração de $\mathrm{Ca}+\mathrm{Mg}$ presente no solo utilizado (tabela 1) é superior ao valor mencionado por estes autores, portanto, possivelmente, justificando a ausência de respostas à omissão desses nutrientes.

As plantas cultivadas na presença de adubação completa apresentaram, aparentemente, desenvolvimento normal. Dentre as omissões de nutrientes (tabela 2) a de $\mathrm{P}$, independente do horizonte do solo, foi a que mais limitou o desenvolvimento da grápia quanto a retenção de folhas. Além disso, foi o tratamento que mais rápido afetou o crescimento (dados não apresentados), sendo que, aos 48 dias do período experimental, diferenciou-se das demais 
Tabela 2 - Efeito da adubação e do solo (Podzólico vermelho amarelo) no crescimento da grápia (Apuleia leiocarpa), aos 165 dias de cultivo, em altura da parte aérea (Alt), diâmetro do caule (Diam), número de nós ( $\mathrm{n}^{\circ}$ nós), número de folhas ( ${ }^{\circ}$ fol), comprimento total do sistema radicular (compSR), massa seca da parte aérea (msPA), massa seca de raiz (msR), massa seca total da planta $(\mathrm{msT})$ e relação da massa seca de raiz/parte aérea (rel R/PA). Os valores referem-se à média dos horizontes $\mathrm{A}$ e $\mathrm{B}$. UFSM, Santa Maria, 1998.

\begin{tabular}{|c|c|c|c|c|c|c|c|c|c|}
\hline Adubação & $\begin{array}{l}\text { Alt } \\
(\mathrm{cm})\end{array}$ & $\begin{array}{l}\text { Diam } \\
(\mathrm{mm})\end{array}$ & $\begin{array}{c}\mathrm{n}^{\circ} \text { nos } \\
\text { (un) }\end{array}$ & $\begin{array}{l}\mathrm{N}^{\circ} \text { fol } \\
\text { (un) }\end{array}$ & $\begin{array}{l}\text { CompSR } \\
(\mathrm{cm})\end{array}$ & $\begin{array}{c}\mathrm{msPA} \\
(\mathrm{g})\end{array}$ & $\begin{array}{c}\mathrm{msR} \\
(\mathrm{g})\end{array}$ & $\begin{array}{c}\mathrm{msT} \\
(\mathrm{g})\end{array}$ & $\mathrm{rel} \mathrm{R/PA}$ \\
\hline COMP & $44,24 a^{*}$ & $4,24 a$ & $17,50 \mathrm{a}$ & $15,25 \mathrm{a}$ & $1456,07 \mathrm{ab}$ & $3,18 \mathrm{a}$ & $1,61 \mathrm{a}$ & $4,80 \mathrm{a}$ & $0,52 \mathrm{c}$ \\
\hline$-\mathrm{N}$ & $20,69 \mathrm{~cd}$ & $2,51 \mathrm{c}$ & $11,50 \mathrm{bc}$ & $7,75 \mathrm{c}$ & $395,46 \mathrm{~cd}$ & $1,00 \mathrm{~cd}$ & $0,73 \mathrm{~b}$ & $1,74 \mathrm{bc}$ & $1,00 \mathrm{~b} \mathrm{c}$ \\
\hline$-\mathrm{P}$ & $11,63 \mathrm{~d}$ & $1,64 \mathrm{~d} e$ & $9,00 \mathrm{~cd}$ & $4,50 \mathrm{de}$ & $420,70 \mathrm{~cd}$ & $0,18 \mathrm{~d}$ & $0,17 \mathrm{~b}$ & $0,36 \mathrm{c}$ & $1,07 \mathrm{~b} c$ \\
\hline$-\mathrm{K}$ & $31,47 \mathrm{~b} \mathrm{c}$ & $3,01 \mathrm{~b} \mathrm{c}$ & $12,75 \mathrm{~b}$ & $10,50 \mathrm{~b}$ & $728,70 \mathrm{bcd}$ & $1,82 \mathrm{bc}$ & $0,79 \mathrm{~b}$ & $2,63 \mathrm{~b}$ & $0,60 \mathrm{c}$ \\
\hline$-\mathrm{Ca}$ & $40,11 \mathrm{ab}$ & $4,22 \mathrm{a}$ & $16,12 a$ & $12,75 \mathrm{ab}$ & $1575,24 \mathrm{a}$ & $3,30 \mathrm{a}$ & $1,67 \mathrm{a}$ & $4,97 \mathrm{a}$ & $0,57 \mathrm{c}$ \\
\hline$-\mathrm{Mg}$ & $46,11 \mathrm{a}$ & $4,14 \mathrm{a}$ & $17,223 \mathrm{a}$ & $13,75 \mathrm{a}$ & $1370,19 \mathrm{ab}$ & $3,62 \mathrm{a}$ & $1,95 \mathrm{a}$ & $5,57 \mathrm{a}$ & $0,56 \mathrm{c}$ \\
\hline$-\mathrm{S}$ & $26,81 \mathrm{c}$ & $2,43 \mathrm{~cd}$ & $13,12 \mathrm{~b}$ & $6,00 \mathrm{~cd}$ & $578,60 \mathrm{~cd}$ & $0,87 \mathrm{dc}$ & $0,54 \mathrm{~b}$ & $1,41 \mathrm{~b} \mathrm{c}$ & $1,39 \mathrm{ab}$ \\
\hline - MICRO & $43,91 \mathrm{a}$ & $3,57 \mathrm{ab}$ & $16,75 a$ & $13,25 \mathrm{a}$ & $937,50 \mathrm{abc}$ & $2,93 \mathrm{a}$ & $1,80 \mathrm{a}$ & $4,73 \mathrm{a}$ & $0,61 \mathrm{c}$ \\
\hline TEST & $9,71 \mathrm{~d}$ & $1,36 \mathrm{e}$ & $6,87 \mathrm{~d}$ & 3,25 e & $148,75 \mathrm{~d}$ & $0,10 \mathrm{~d}$ & $0,15 \mathrm{~b}$ & $0,25 \mathrm{c}$ & $1,74 \mathrm{a}$ \\
\hline \multicolumn{10}{|l|}{ Horizonte } \\
\hline A & $36,92 \mathrm{a}$ & $3,38 \mathrm{a}$ & $14,58 \mathrm{a}$ & $10,92 \mathrm{a}$ & $818,38 \mathrm{a}$ & $2,43 a$ & $1,16 \mathrm{a}$ & $3,60 \mathrm{a}$ & $0,69 \mathrm{~b}$ \\
\hline $\mathrm{B}$ & $24,25 \mathrm{~b}$ & $2,65 \mathrm{~b}$ & $12,28 \mathrm{~b}$ & $7,97 \mathrm{~b}$ & $804,11 \mathrm{a}$ & $1,34 \mathrm{~b}$ & $0,93 \mathrm{~b}$ & $2,28 \mathrm{~b}$ & $1,11 \mathrm{a}$ \\
\hline
\end{tabular}

* Tratamentos com médias não ligadas por mesma letra, na vertical, diferem pelo teste de Tukey em nível de 5\% de probabilidade.

deficiências, demonstrando o menor crescimento quanto à altura, número de folhas e diâmetro do caule. Já aquelas submetidas à deficiência em $\mathrm{N}$ no horizonte A, mostraram paralisação do crescimento e clorose nas folhas mais velhas após o $3^{\circ}$ mês de condução do experimento (dados não apresentados). Enquanto aquelas, no horizonte B, praticamente não se desenvolveram desde o $2^{\circ}$ mês pela acentuada deficiência de $\mathrm{N}$, adquirindo coloração clorótica em toda a planta.

A omissão de $\mathrm{S}$ também afetou significativamente o desenvolvimento da parte aérea e do sistema radicular das plantas. A aplicação de $30 \mathrm{mgkg}^{-1}$ de $\mathrm{S}$ na adubação completa sugere ter proporcionado desenvolvimento satisfatório às plantas. NOVAIS et al. (1992) salientaram que doses entre 20 e $40 \mathrm{mgkg}^{-1}$ de $\mathrm{S}$ são ideais para o desenvolvimento inicial de Eucalyptus grandis e a aplicação de doses superiores a $40 \mathrm{mgkg}^{-1}$ não proporcionou ganhos consideráveis.

$\mathrm{Na}$ ausência de K, as plantas apresentaram clorose leve nas folhas mais velhas (dados não apresentados). Nas cultivadas no horizonte B, houve paralisação do desenvolvimento após o $3^{\circ}$ mês do experimento, enquanto que no horizonte $\mathrm{A}$, as plantas continuaram se desenvolvendo normalmente até os 165 dias.

A omissão de $\mathrm{P}, \mathrm{S}$ e $\mathrm{N}$, juntamente ao tratamento testemunha, apresentaram as menores médias para todos os parâmetros de crescimento avaliados, em ambos horizontes de solo (tabela 2). Demonstrando, assim, que o $\mathrm{P}, \mathrm{S}$ e $\mathrm{N}$ são os nutrientes mais limitantes no solo Podzólico vermelho amarelo para o desenvolvimento inicial da grápia. BRAGA et al. (1995), trabalhando com quatro espécies florestais, observaram que as espécies Acacia mangiun e Platycyamus regnellii mostraram-se particularmente exigentes em P, N e S. Já a Aspidosperma polyneuron mostrou-se exigente em P, K e S e a espécie Tibouchina granulosa respondeu à adubação com todos os macro e micronutrientes. DIASS et al. (1994), trabalhando com Acacia mangiun, observaram que a omissão de $\mathrm{N}$ foi o que mais afetou a produção de matéria seca da parte aérea, seguido pela omissão de $\mathrm{S}$ e P.

A relação entre a massa seca de raízes e da parte aérea (R/PA) foi principalmente afetada pela omissão de $\mathrm{S}, \mathrm{P}$ e N (tabela 2 ). A relação R/PA é maior em ambiente de baixa fertilidade, podendo ser considerada uma estratégia da planta para retirar o máximo de nutrientes daquela condição (Clarson apud BRAGA et al., 1995). Segundo DANIEL et al. (1997), o valor ideal desta partição para plantas em desenvolvimento normal está próximo a 0,5. Valores próximos a este foram conseguidos no presente experimento apenas nas plantas que receberam adubação completa e na omissão de $\mathrm{K}, \mathrm{Ca}, \mathrm{Mg}$ e micronutrientes, possivelmente, porque os teores já existentes no solo foram suficientes às plantas durante o período experimental.

A omissão de N, P, S, K e micronutrientes, em menor proporção para os dois últimos tratamentos, afetou negativamente a produção de massa seca das raízes e o comprimento total do sistema 
radicular, provavelmente pela inibição do desenvolvimento das raízes secundárias e de outras ordens menores. HIROCE et al. (1990) observaram fato semelhante em Cyperus malaccensis, em que a ausência de $\mathrm{K}$ afetou drasticamente a produção de massa seca do sistema radicular. Fato também comprovado por BRAGA et al. (1995), que constataram ser o K o nutriente mais importante para a produção de raízes secundárias e o $\mathrm{S}$ para o crescimento da raiz principal.

\section{Teores de nutrientes nos tecidos:}

Entre os tratamentos, em ambos horizontes, observou-se que a omissão do $\mathrm{S}$ resultou em maiores teores de $\mathrm{N}$ nos diferentes órgãos da planta (tabela 3), demonstrando a estreita relação entre $\mathrm{N} \mathrm{e}$

Tabela 3 - Teores de nutrientes nos tecidos da folha, caule e raiz de plantas de grápia (Apuleia leiocarpa) cultivadas no horizonte A e B do solo Podzólico vermelho amarelo e submetidos a diferentes tratamentos de adubação. Os valores referem-se à média aritmética de no mínimo duas análises. UFSM, Santa Maria, 1998.

\begin{tabular}{|c|c|c|c|c|c|c|c|c|c|c|c|c|c|c|c|c|c|c|}
\hline \multirow[b]{4}{*}{ TRAT } & \multicolumn{9}{|c|}{ HORIZONTE A } & \multicolumn{9}{|c|}{ HORIZONTE B } \\
\hline & \multicolumn{9}{|c|}{ FOLHA } & \multicolumn{9}{|c|}{ FOLHA } \\
\hline & \multicolumn{5}{|c|}{$\%$} & \multicolumn{4}{|c|}{ ppm } & \multicolumn{5}{|c|}{$\%$} & \multicolumn{4}{|c|}{ ppm } \\
\hline & $\mathrm{N}$ & $\mathrm{P}$ & $\mathrm{K}$ & $\mathrm{Ca}$ & $\mathrm{Mg}$ & $\mathrm{Zn}$ & $\mathrm{Mn}$ & $\mathrm{Cu}$ & $\mathrm{Fe}$ & $\mathrm{N}$ & $P$ & $\mathrm{~K}$ & $\mathrm{Ca}$ & $\mathrm{Mg}$ & $\mathrm{Zn}$ & $\mathrm{Mn}$ & $\mathrm{Cu}$ & $\mathrm{Fe}$ \\
\hline COMP & 2,66 & 0,18 & 1,19 & 0,68 & 0,43 & 45 & 444 & 3,52 & 295 & 2,22 & 0,12 & 0,93 & 0,70 & 0,37 & 62 & 159 & 3,07 & 129 \\
\hline$-\mathrm{N}$ & 2,01 & 0,16 & 1,14 & 0,89 & 0,41 & 101 & 306 & 1,17 & 307 & 1,60 & 0,11 & 1,14 & 0,64 & 0,40 & 40 & 154 & 1,27 & 96 \\
\hline$-P$ & 3,61 & 0,05 & 1,70 & 0,60 & 0,39 & 56 & 96 & 1,70 & 592 & 2,04 & 0,04 & 1,08 & 0,56 & 0,30 & - & - & - & - \\
\hline$-K$ & 3,88 & 0,18 & 0,62 & 0,65 & 0,51 & 57 & 233 & 0,99 & 140 & 2,61 & 0,08 & 0,45 & 0,62 & 0,50 & 40 & 193 & 0,42 & 110 \\
\hline$-\mathrm{Ca}$ & 2,57 & 0,13 & 1,08 & 0,71 & 0,44 & 51 & 313 & 0,75 & 139 & 3,16 & 0,18 & 0,77 & 0,74 & 0,42 & 56 & 141 & 1,29 & 122 \\
\hline$-\mathrm{Mg}$ & 3,50 & 0,18 & 1,15 & 0,65 & 0,38 & 63 & 345 & 0,74 & 166 & 2,55 & 0,12 & 0,92 & 0,65 & 0,40 & 69 & 156 & 0,86 & 126 \\
\hline$-S^{\circ}$ & 3,84 & 0,23 & 1,31 & 0,76 & 0,48 & 52 & 288 & 0,96 & 199 & - & - & - & - & - & - & - & - & - \\
\hline -MICRO & 3,79 & 0,13 & 1,20 & 0,67 & 0,46 & 40 & 338 & 2,20 & 232 & 3,21 & 0,13 & 1,28 & 0,62 & 0,46 & 57 & 163 & 1,30 & 185 \\
\hline \multirow[t]{3}{*}{ TEST } & & - & - & - & - & - & - & - & - & - & - & - & - & - & - & - & - & - \\
\hline & \multicolumn{9}{|c|}{ CAULE } & \multicolumn{9}{|c|}{ CAULE } \\
\hline & \multicolumn{5}{|c|}{$\%$} & \multicolumn{4}{|c|}{ ppm } & \multicolumn{5}{|c|}{$\%$} & \multicolumn{4}{|c|}{ ppm } \\
\hline COMP & 1,49 & 0,05 & 0,87 & 0,43 & 0,23 & 54 & 89 & 1,00 & 70 & 1,39 & 0,05 & 0,52 & 0,46 & 0,23 & 29 & 48 & 1,14 & 139 \\
\hline$-\mathrm{N}$ & 0,65 & 0,09 & 0,63 & 0,63 & 0,16 & 34 & 70 & 1,89 & 103 & 0,24 & 0,05 & 0,28 & 0,32 & 0,16 & 44 & 53 & 1,55 & 108 \\
\hline$-P$ & 1,24 & 0,04 & 0,41 & 0,70 & 0,31 & - & - & - & - & 0,46 & 0,04 & 0,38 & 0,30 & 0,17 & - & - & - & - \\
\hline$-\mathrm{K}$ & 1,70 & 0,08 & 0,44 & 0,41 & 0,31 & 61 & 65 & 1,40 & 99 & 1,30 & 0,04 & 0,33 & 0,47 & 0,22 & 73 & 63 & 1,22 & 50 \\
\hline$-\mathrm{Ca}$ & 1,40 & 0,04 & 0,52 & 0,46 & 0,22 & 52 & 27 & 1,00 & 47 & 1,24 & 0,04 & 0,82 & 0,52 & 0,22 & 65 & 43 & 1,80 & 117 \\
\hline$-\mathrm{Mg}$ & 1,83 & 0,04 & 0,55 & 0,46 & 0,23 & 55 & 75 & 1,85 & 60 & 0,70 & 0,04 & 0,74 & 0,42 & 0,21 & 52 & 21 & 0,85 & 63 \\
\hline$-S^{\circ}$ & 2,32 & 0,07 & 0,47 & 0,62 & 0,20 & 57 & 40 & 1,60 & 116 & - & - & - & - & - & - & - & - & - \\
\hline -MICRO & 1,12 & 0,06 & 0,60 & 0,42 & 0,24 & 90 & 85 & 1,07 & 123 & 1,26 & 0,03 & 0,63 & 0,37 & 0,28 & 63 & 31 & 1,27 & 53 \\
\hline TEST & 0,98 & 0,08 & 0,29 & 0,52 & 0,22 & - & - & - & - & 0,23 & 0,05 & 0,27 & 0,27 & 0,10 & - & - & - & - \\
\hline & \multicolumn{9}{|c|}{ RAIZ } & \multicolumn{9}{|c|}{ RAIZ } \\
\hline & \multicolumn{5}{|c|}{$\%$} & \multicolumn{4}{|c|}{ ppm } & \multicolumn{5}{|c|}{$\%$} & \multicolumn{4}{|c|}{ ppm } \\
\hline COMP & 1,12 & 0,06 & 0,49 & 0,18 & 0,18 & 113 & 17 & 4,97 & 391 & 1,05 & 0,04 & 0,49 & 0,18 & 0,20 & 105 & 19 & 3,12 & 419 \\
\hline$-\mathrm{N}$ & 0,18 & 0,07 & 0,55 & 0,22 & 0,18 & 54 & 42 & 1,84 & 290 & 0,52 & 0,03 & 0,42 & 0,12 & 0,15 & 147 & 60 & 7,81 & 854 \\
\hline$-P$ & 2,01 & 0,04 & 0,36 & 0,14 & 0,14 & 106 & 59 & 2,78 & 285 & - & - & - & - & - & 142 & 35 & 5,50 & 678 \\
\hline$-K$ & 1,56 & 0,06 & 0,22 & 0,12 & 0,15 & 98 & 158 & 4,40 & 370 & 1,2 & 0,04 & 0,22 & 0,12 & 0,43 & 77 & 36 & 8,66 & 945 \\
\hline$-\mathrm{Ca}$ & 1,33 & 0,04 & 0,44 & 0,17 & 0,17 & 83 & 81 & 2,98 & 233 & 1,05 & 0,05 & 0,44 & 0,17 & 0,17 & 68 & 23 & 2,98 & 301 \\
\hline$-\mathrm{Mg}$ & 1,65 & 0,05 & 0,38 & 0,24 & 0,24 & 115 & 162 & 4,83 & 527 & 1,22 & 0,05 & 0,44 & 0,27 & 0,22 & 73 & 23 & 2,69 & 464 \\
\hline$-S$ & 2,03 & 0,07 & 0,44 & 0,16 & 0,16 & 111 & 98 & 6,67 & 503 & 2,20 & 0,07 & 0,44 & 0,14 & 0,17 & - & - & - & - \\
\hline -MICRO & 2,01 & 0,05 & 0,49 & 0,29 & 0,39 & 95 & 123 & 2,84 & 391 & 1,52 & 0,04 & 0,47 & 0,13 & 0,14 & 62 & 32 & 2,84 & 401 \\
\hline TEST & 1,24 & 0,04 & 0,44 & 0,15 & 0,15 & 114 & 54 & 2,45 & 752 & 1,09 & 0,05 & 0,38 & 0,09 & 0,12 & - & - & - & - \\
\hline
\end{tabular}

Ciência Rural, v. 29, n. 2, 1999. 
S no metabolismo, evidenciada por BRUNOLD \& SUTER (1994). DIAS et al. (1994) constataram esse mesmo fenômeno em Acacia mangiun. Esses autores também observaram, semelhante ao registrado para a grápia (tabela 3 ), que o teor de $\mathrm{P}$ aumentou com a omissão do S. Este comportamento pode ser atribuído, em parte, à redução na produção de massa seca do tratamento com omissão do S.

Em ambos horizontes, a concentração de $\mathrm{Fe}, \mathrm{Cu}$ e $\mathrm{Zn}$ foram maiores nas raízes que no caule e folhas. Contudo, salienta-se mais a presença da alta concentração de $\mathrm{Fe}(73,8 \%$ do encontrado na planta) nas raízes das plantas cultivadas no horizonte $\mathrm{B}$, indistintamente do tratamento de adubação; fato que pode indicar a ocorrência de distúrbios fisiológicos. Observou-se, também, que a concentração de $\mathrm{K}$ foi menor nas raízes e folhas de plantas cultivadas no horizonte B (Tabela 3). Segundo Tanaka et al. apud MENGEL \& KIRKBY (1979), a toxicidade de Fe, em várias áreas de cultivo de arroz, é freqüentemente associada à deficiência de K. O Mn foi consideravelmente mais concentrado nas folhas. Quanto aos macronutrientes, observou-se que o N, P, K, Ca e $\mathrm{Mg}$ apresentam-se mais concentrados nas folhas (50\% daquele da planta).

Considerando-se os teores adequados dos nutrientes $\mathrm{N}, \mathrm{P}, \mathrm{K}, \mathrm{Ca}, \mathrm{Mg}, \mathrm{Zn}, \mathrm{Cu}, \mathrm{Fe}$ e $\mathrm{Mn}$ nas folhas, para as espécies Araucária, Eucalipto e Pinus, apresentados por MALAVOLTA et $\boldsymbol{a l}$. (1997), verificou-se que a grápia, quando cultivada no horizonte A e submetida à adubação completa ou na omissão isolada de $\mathrm{Ca}, \mathrm{Mg}, \mathrm{K}$ e micronutrientes, apresentou concentração desses nutrientes dentro da faixa recomendada. Portanto, demonstrando que os teores já existentes no solo foram suficientes às plantas durante o período experimental. Por outro lado, quando submetida à condição de solo do horizonte $\mathrm{B}$, observam-se teores inadequados de $\mathrm{N}$ e $\mathrm{P}$, principalmente. Justificando a diferença de acumulação de biomassa entre as plantas crescidas nesses solos e, também, indicando uma limitação potencial do horizonte $\mathrm{B}$ do solo Podzólico vermelho amarelo para sustentar o cultivo da grápia. Acrescentam-se a esse fato dois outros fatores negativos: (i) a alta concentração de $\mathrm{Al}^{+3}$ e a baixa concentração de P no horizonte B (Tabela 1) e (ii) a presença de um sistema radicular pouco denso dessa espécie, se comparado a outras espécies não florestais, tais como a Pfaffia glomerata (NICOLOSO et al.,1999). A soma desses fatores pode ser consideravelmente danosa, principalmente, a nutrição fosfatada em períodos de deficiência hídrica, considerando-se que o fósforo inorgânico tem como mecanismo principal de suprimento às raízes a difusão (MALAVOLTA et al., 1997).

\section{CONCLUSÕES}

A omissão isolada de $\mathrm{Ca}, \mathrm{Mg}$ e dos micronutrientes $\mathrm{Zn}, \mathrm{Cu}, \mathrm{Fe}, \mathrm{Mn}$ da adubação não afeta o crescimento da grápia em altura, diâmetro do caule, número de nós, número de folhas e a produção da massa seca, bem como a concentração desses elementos nos tecidos da planta, demonstrando que os teores naturais do solo são suficientes.

A altura da planta, número de nós, número de folhas, comprimento do sistema radicular, produção de massa seca e a concentração de nutrientes diminuem acentuadamente pela omissão de $\mathrm{P}$, $\mathrm{S}, \mathrm{N}$ e K da adubação, tanto no horizonte A como no B do solo Podzólico vermelho-amarelo.

\section{REFERÊNCIAS BIBLIOGRÁFICAS}

BRAGA, F.A.M., VALE, F.R., VENTORIM, N., et al. Exigências nutricionais de quatro espécies florestais. Revista Árvore, Viçosa, v. 19, n. 1, p. 18-31, 1995.

BRUNOLD, C., SUTER, M. Regulation of sulfate assimilation by nitrogen in the duckweed Lemna minor L. Plant Phisiology, Baltimore, n. 76, p. 579-583. 1984.

CEVENÁ, M., KULHAVÝ, J. Effect of aluminiun on the roots of Picea abies seedlings. In: Plants roots and their environment - Developments in agricultural and managedforest ecology; $24^{\circ}$. Amsterdam, 1991. $649 \mathrm{p}$.

DANIEL, O., VITORINO, A.C.T., ALOVISI, A.A., et al. Aplicação de fósforo em mudas de Acacia mangium Willd Revista Árvore, Viçosa, v. 21, n. 2, p. 163-168, 1997.

DIAS, L.E., FARIA, S.M., FRANCO, A.A. Crescimento de mudas de Acacia mangium Willd em resposta à omissão de macronutrientes. Revista Árvore, Viçosa, v. 18, n. 2, p. 123131,1994

FEITOSA, C.I., HIROCE, R., BENATTI, R.J. Omissão de macronutrientes em rami. Bragantia, Campinas, v. 51, n. 2, p. 425-429, 1992.

HERINGER, E.P., FERREIRA, M.B. Árvores úteis da Região Geo-Econômica do Distrito Federal. Cerrado, Brasília, v. 19, p. 20-24, 1973.

HIROCE, R., FEITOSA, C.T., BENATTI, R.J. Omissão de macronutrientes em Junco. Bragantia, Campinas, v. 49, n. 2 , p. $425-429,1990$

MALAVOLTA, E. Potássio, magnésio e enxofre nos solos e culturas brasileiras. Piracicaba: POTAFOS, 1984. 91 p. Boletim Técnico, 4.

MALAVOlTA, E., VITTI, G.C., OLIVEIRA, S.A. Avaliação do estado nutricional das plantas: princípios e aplicações. Piracicaba: POTAFOS, 1997, 319 p.

MARTINEZ, H.E.P., HAAG, H.P., MORAES, M.L.T Micronutrientes em Pinus caribae Morelet, III. Níveis internos de $\mathrm{Fe}$, Mn e $\mathrm{Zn}$ sob suficiência e sob omissão. Pesquisa Agropecuária Brasileira, Brasília, v. 27, n. 9, p. 1339-1353, 1992. 
MENGEL, K., KIRKBY, E.A. Principles of plant nutrition. International Potash Institute. Worblaufer-Bern, 1979. 579p.

NOVAIS, R.F, BARROS, N.F., NEVES, J.C.L. Nutrição mineral do Eucalipto. In: BARROS, N.F., NOVAIS, R.F. Relação solo-eucalipto, Viçosa: UFV, 1992, cap. 2, p. 25-98

NICOLOSO, F.T., GARLET, A., ZANCHETTI, F., et al. . Efeito de métodos de escarificação na superação da dormência de sementes e de substratos na germinação e no desenvolvimento de sementes de grápia (Apuleia leiocarpa). Ciência Rural, Santa Maria, v. 27, n. 3, p. 419-424, 1997.

NICOLOSO, F.T, FORTUNATO, R.P., FOGAÇA, M.A.F. Influência da posição da estaca no ramo sobre o enraizamento de estacas de Pfaffia glomerata (Spreng.) Pederson, em dois substratos. Ciência Rural, Santa Maria, v. 29, n. 2, p. 277283,1999

SIMÕES, J.W. Efeito da omissão de nutrientes na alimentação mineral do Pinheiro do Paraná Araucaria angustifolia (BERT.) O. KTZE cultivada em vaso. In: II CONGRESSO FLORESTAL BRASILEIRO, Curitiba, 1973. Anais... Curitiba: 1973. p. 112-121.

TEDESCO, M.S., GIANELlO, C., BISSANI, C.A., et al. Análise de solo, plantas e outros materiais. Porto Alegre: Universidade Federal do Rio Grande do Sul, 1995. 174 p., Boletim Técnico, 5.

TENNANT, D.A. Test of modified line intersect method of estimating root lenght. The Journal of Ecology, v. 63, n. 3, p. 995-1001, 1975

Ciência Rural, v. 29, n. 2, 1999. 